\title{
The comparison of two methods in cervical smear screening - which method is better for smear adequacy rates?
}

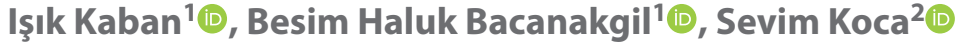 \\ 'Istanbul Training and Research Hospital Gynecology And Obstetric Department Istanbul, Turkey \\ ${ }^{2}$ Istanbul Training and Research Hospital Pathology Department Istanbul, Turkey
}

\begin{abstract}
Objectives: In the cervical smear screening test as a sample collection method for liquid-based thin layer cytology, classically the collecting device is placed into a liquid fixative solution and vigorously swirled or rotated ten times in the solution and the collection device is removed from the solution. In this study, a plastic smear brush was used as the collection device. After the cervical cell sample was obtained, the smear brush was detached from the stick and left in the solution and given to the laboratory. Our aim in the study is to examine whether smear inadequacy rates have decreased with the method used in the study compared to the classical method.
\end{abstract}

Material and methods: While the classical technique which the collecting device is placed into a solution and mixed and removed from the solution is defined as Method 1. The technique used in the study was defined as Method 2 . The cervical smear screening test results obtained by two different methods in two consecutive time periods were analyzed. The two methods were compared using chi-square test in terms of smear inadequacy.

Results: A total of 2129 test results, including 1129 smears in Method 1 and 1000 smears in Method 2 were examined. The mean ages of the patients tested in both methods were similar ( $36 \pm 6.1$ and $37 \pm 6.7$ ). Abnormal test result rate was similar for Method 1 and Method 2 (5.8\% vs 4.9\%, respectively). The inadequate sample rate was higher in Method 1 than Method 2 ( $8.3 \%$ vs $2.1 \%$, respectively).

Conclusions: The study showed that leaving the smear brush in the solution is a better way to reduce the inadequacy sample rates. This result may guide clinicians about smear techniques.

Key words: cervical smear; inadequacy rates; liquid-based cytology; thin prep

Ginekologia Polska 2021; 92, 5: 335-338

\section{INTRODUCTION}

Cervical cancer is the second most common cause of cancer death in women worldwide [1]. The screening test of the cervical smear (Pap smear) has led to a dramatic decrease in the incidence of cervical cancer in the past 60 years [2]. Nevertheless, according to the 2020 cancer report of the World Health Organization (WHO), more than half a million women are diagnosed with cervical cancer worldwide annually [3].

There are two main cervical smear techniques, the conventional technique and the liquid-based technique. These have been described as screening tests for cervical invasive or preinvasive lesions [4]. In the conventional Pap smear technique, the cervical swab sample taken with a brush is involved in direct transfer to the microscope slide for evaluation. This method has an inadequacy rate ranging from $5 \%$ to $25 \%$ according to studies [5-9]. Causes such as drying in the air, bad fixation, blood, inflammation, thick areas and foreign body are the causes of smear failure for conventional pap smear. The liquid-based technique was approved by the Food and Drug Administration in May 1996 as an alternative to traditional conventional smear [10]. Within the last two decades, SurePath and ThinPrep (both liquid-based cytology (LBC) tests] have replaced conven- 
tional cytology (CC) as the primary test method in cervical cancer screening programs [11]. With ThinPrep, a cervical specimen is collected using a Cervix Brush, and the brush is rinsed in a vial with a methanol based preservative fluid. Cells are released by pushing the brush to the bottom, forcing the bristles apart, and swirling the brush into the fluid. Subsequently, the brush is discarded. At the laboratory, cells are isolated from the fluid by vacuum filtration and are transferred to the slide using air pressure for adherence [11]. The main cause of smear inadequacy for this technique is the scarcity of cervical squamous cells in the smear sample. According to general recommendation for thin prep, the collecting device is placed into a liquid fixative solution and vigorously swirled or rotated ten times in the solution, then the brush is discarded [11, 12]. We identified this method as "Method 1" in our study. Instead of removing the smear brush, we left the brush in the solution in the smear box and identified this as "Method 2". Our goal was to examine whether this was effective in reducing smear inadequacy. There are many studies about cervical cytology sample collection devices [13-15]. However, we did not find a study in literature comparing leaving the brush in solution with removing the brush.

\section{MATERIAL AND METHODS}

Ethics committee approval was received for this study from the Istanbul Training and Research Hospital (number: 1880 date: 28 June 2019). In this study, two consecutive cross-sectional cohort smear results were examined. Within the last two decades, SurePath and ThinPrep have replaced conventional cytology as the primary test method in cervical cancer screening programs, so we prefer the liquid base cytology in our hospital. In our clinic, Method 1 is used as recommended in the literature. For this study, smear screening test was performed with Method 2 for a specified time period. The study was designed as prospective cross-sectional observational. Samples were obtained from patients who applied to the gynaecology outpatient clinic and required routine smear screening testing. With ThinPrep, a cervical specimen is collected using a Cervix Brush, and the brush is rinsed in a vial with a methanol-based preservative fluid (PreservCyt Solution 20 ml, Hologic Inc., Marlborough, USA). Cells are released by pushing the brush to the bottom, forcing the bristles apart, and swirling the brush into the fluid. Subsequently, the brush is discarded. At the laboratory, cells are isolated from the fluid via vacuum filtration and are transferred to the slide using air pressure for adherence with (ThinPrep 2000 Processor, Hologic Inc., Marlborough, MA, USA). Cell samples for cervical cytology were obtained during the speculum examination and a swab sample from the uterine cervix was obtained using cervical brush. Next we rotated the brush 180 degrees to obtain a sample if

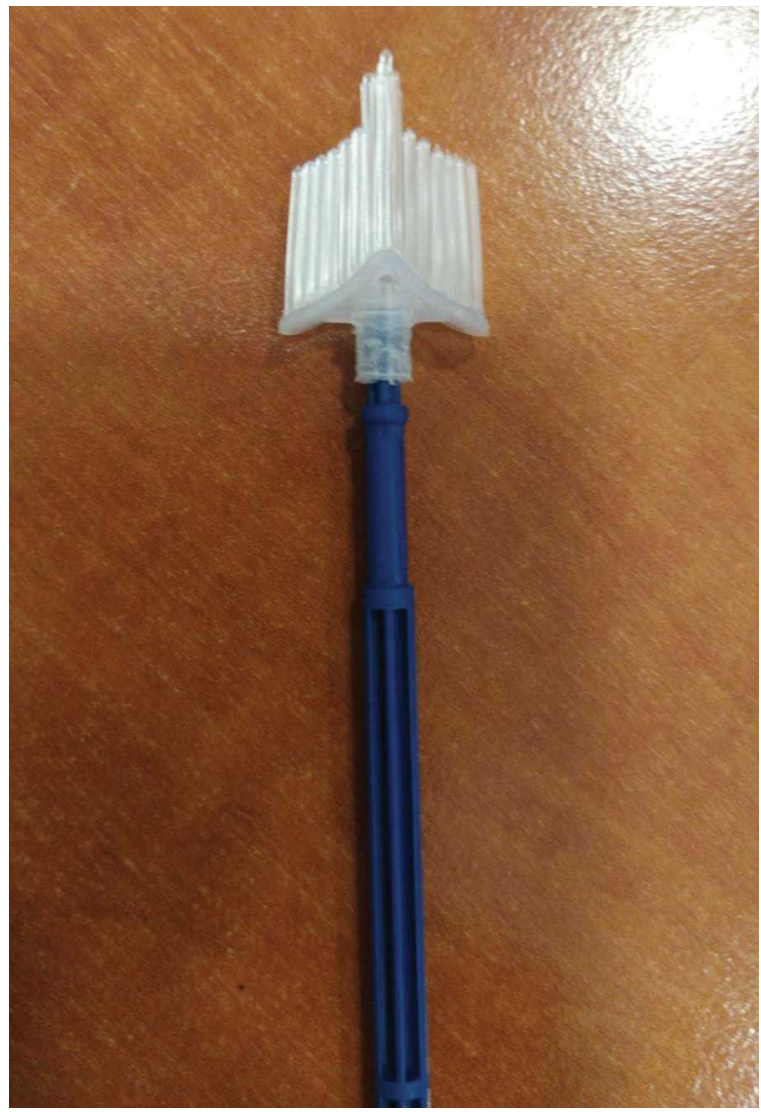

Figure 1. Disposable plastic smear brush

there was no inflamed discharge, bleeding, gross tumor. Gel lubricant on the speculum or on an examiner's hand before performing a Pap test is commonly thought to interfere with the results of cervical cytology. So no lubricants were used during sampling. A sterile medical disposable vaginal cervical cytology sampling brush was used as a product for sampling (Fig. 1). The smear brush was vigorously swirled or rotated ten times in the solution and then it was then removed (Method 1). In Method 2, once disconnected from the handles, the brush heads were deposited into the vial containing liquid preservative (not removed). The test results obtained with both methods were examined by our pahtology clinic.

Statistical analysis was performed using the IBM SPSS Statistics 21.0 software. Group differences were analyzed using the chi-square test. A p-value $<0.05$ was considered to indicate statistical significance.

\section{RESULTS}

Initially, Method 1 was performed in gynaecology performing rooms for three months and then the second stage of the study was carried out with Method 2 in gynaecology performing rooms for three months in our hospital. 
Table 1. Comparison of smear screening methods

\begin{tabular}{|l|c|c|c|}
\hline Feature & Method 1 & Method 2 & P value \\
\hline Number & 1129 & 1000 & 0.654 \\
\hline Age [years], mean \pm SD; min-max & $36 \pm 6.1 ; 21-67$ & $37 \pm 6.7 ; 19-63$ & 0.001 \\
\hline Inadequacy of smear & $94(8.3 \%)$ & $21(2.1 \%)$ & 0.186 \\
\hline Abnormal cytological findings, $n(\%)$ & $66(5.8 \%)$ & $49(4.9 \%)$ & \\
ASC-US & 37 & 24 & \\
AG-US & 1 & - & \\
LGSIL & 18 & 4 & \\
HG-SIL & 9 & 2 & \\
ASC-H & 1 & 2 & \\
\hline
\end{tabular}

SD — standard deviation; ASC-US — atypical squamous cells of undetermined significance; AG-US — atypical glandular cells of undetermined significance; LGSIL — low grade squamous intraepithelial lesions; HGSIL — high grade squamous intraepithelial lesion; ASC-H — atypical squamous cell cannot exclude HSIL

A total of 2129 test results were examined. Of these, 1129 were obtained with Method 1 and 1000 with Method 2. The mean ages of the patients tested in both methods were similar ( $36 \pm 6.1$ and $37 \pm 6.7$ ) (Tab. 1). The rate of abnormal test results were $5.8 \%$ in Method 1 and 4.9 in Method 2. Details of the abnormal results are shown in Table 1.

The inadequate smear result rate was $8.3 \%$ in Method 1 and $2.1 \%$ in Method 2. The reason for the inadequacies was the scarcity of cervical squamous cells in the smear sample in all cases.

\section{DISCUSSION}

In this study, two different methods for sample collection in the liquid based cervical cancer screening test were compared in terms of smear inadequacy. As the first method, the smear brush is immersed in the solution, swirled and then removed (Method 1). Secondly, the smear brush stick is left in the solution, which is the method that was tested in this study (Method 2). The results support that Method 2 is more advantageous than Method 1 in terms of smear adequacy rates. According to the results of more than two thousand smears in this study, Method 2 was calculated to have four times lower smear inadequacy rate ( $8.3 \%$ vs $2.1 \%$ ).

Evaluation of specimen adequacy is considered by experts to be the most important quality assurance component of the Bethesda system [16]. Satisfactory cervical cytology is defined by the number of squamous cells in the sample. According to this study, leaving the smear brush in solution and delivering it to the pathology laboratory was advantageous in terms of squamous cell number. This method may provide sufficient time for the squamous cell samples taken from the cervix to pass into the solution because the smear brush remains in the solution until the pathologist examination. In the classical method, the smear brush is immersed in solution, shaken, and then removed from the solution. Cervical cell samples must pass into the solution from the smear brush within a period of time for these procedures. Sufficient time for cervical squamous cell pass to the solution may not be given with this method.

Our limitations were that all materials could not be collected from the same obstetrician and also not examined from the same pathologist. It was a result of the large patient numbers of this study.

In conclusion, the study showed that leaving the smear brush in the solution is a better way to reduce the smear rate. These results need to be supported by randomized controlled trials. The results of this study can give clinicians an idea about the smear screening test sample collection method. Clinicians or screening test sample collectors can benefit from the results of this study on the smear screening test sample collection method. Also, when collecting smears again for cases reported as inadequate sample, the method that this study finds advantageous can be used.

\section{Conflict of interests}

The authors declare that they have no conflict of interests.

\section{REFERENCES}

1. Torre LA, Islami F, Siegel RL, et al. Global Cancer in Women: Burden and Trends. Cancer Epidemiol Biomarkers Prev. 2017; 26(4): 444-457, doi: 10.1158/1055-9965.EPI-16-0858, indexed in Pubmed: 28223433.

2. Masenya M. Liquid based cytology. Obstetrics and Gynaecology Forum. 2011; 21(3), doi: 10.4314/ogf.v21i3.69484.

3. WHO-CancerReport-2020-Global Profile. 2020.

4. McGoogan E, Colgan TJ, Ramzy I, et al. Cell preparation methods and criteria for sample adequacy. International Academy of Cytology Task Force summary. Diagnostic Cytology Towards the 21st Century: An International Expert Conference and Tutorial. Acta Cytol. 1998; 42(1): 25-32, doi: 10.1159/000331532, indexed in Pubmed: 9479321.

5. Karabacak T, Aydın Ö, Düşmez D, et al. Limitation, inadequacy rates and reasons in cervicovaginal smears (2832 cases). Turkish J Pathol. 2001; 18(3): 22-25.

6. Daglı AF, Ozercan MR. Servikal Smear Tarama Programımızda Sınırlılık/Yetersizlik Oranları ve Nedenleri (1322 Olgu). Fırat Med J. 2006; 11(3): 166-169.

7. Celik C, Gezginç K, Toy H, et al. A comparison of liquid-based cytology with conventional cytology. Int J Gynaecol Obstet. 2008; 100(2): 163-166, doi: 10.1016/j.ijgo.2007.07.023, indexed in Pubmed: 17920599.

8. Jeong H, Hong SR, Chae SW, et al. Comparison of Unsatisfactory Samples from Conventional Smear versus Liquid-Based Cytology in Uterine Cervi- 
cal Cancer Screening Test. J Pathol Transl Med. 2017; 51(3): 314-319, doi: 10.4132/jptm.2017.03.17, indexed in Pubmed: 28415159.

9. Singh VB, Gupta N, Nijhawan R, et al. Liquid-based cytology versus conventional cytology for evaluation of cervical Pap smears: experience from the first 1000 split samples. Indian J Pathol Microbiol. 2015; 58(1): 17-21, doi: 10.4103/0377-4929.151157, indexed in Pubmed: 25673585.

10. Guidos B, Selvaggi S. Use of the Thin Prep Pap Test? in clinical practice. Diagnostic Cytopathology. 1999; 20(2): 70-73, doi: 10.1002/(sici)10970339(199902)20:2<70::aid-dc5>3.0.co;2-e.

11. Rozemeijer K, Penning C, Siebers AG, et al. Comparing SurePath, ThinPrep, and conventional cytology as primary test method: SurePath is associated with increased CIN II+ detection rates. Cancer Causes Control. 2016; 27(1): 15-25, doi: 10.1007/s10552-015-0678-1, indexed in Pubmed: 26458884.

12. http://www.thinprep.com/hcp/specimen_collection/brush_spatula. html (25.04.2012)
13. Martin-Hirsch $P$, Jarvis $G$, Kitchener $H$, et al. Collection devices for obtaining cervical cytology samples. Cochrane Database Syst Rev. 2000(2): CD001036, doi: 10.1002/14651858.CD001036, indexed in Pubmed: 10796736.

14. Marchand L, Mundt M, Klein G, et al. Optimal collection technique and devices for a quality pap smear. WMJ. 2005; 104(6): 51-55, indexed in Pubmed: 16218317.

15. Schnippel K, Michelow $\mathrm{P}, \mathrm{Chibwesha} \mathrm{CJ}$, et al. Cost-effectiveness of using the Cervex-Brush (broom) compared to the elongated spatula for collection of conventional cervical cytology samples within a high-burden HIV setting: a model-based analysis. BMC Health Serv Res. 2015; 15 499, doi: 10.1186/s12913-015-1163-y, indexed in Pubmed: 26545585.

16. Wilbur DC, Nayar R, Nayar R, et al. The Pap Test and Bethesda 2014: "The reports of my demise have been greatly exaggerated." (after a quotation from Mark Twain). J Am Soc Cytopathol. 2015; 4(3): 170-180, doi: 10.1016/j.jasc.2015.03.003, indexed in Pubmed: 31051698. 